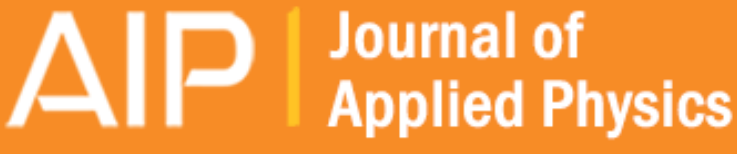

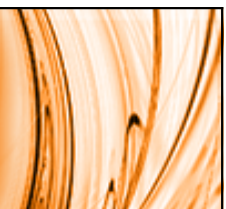

\section{A comparison of numerical simulations and analytical theory of the dynamics of interacting magnetic vortices}

Martin Asmat-Uceda, Xuemei Cheng, Xiao Wang, David J. Clarke, Oleg Tchernyshyov, and Kristen S. Buchanan

Citation: Journal of Applied Physics 117, 123916 (2015); doi: 10.1063/1.4916610

View online: http://dx.doi.org/10.1063/1.4916610

View Table of Contents: http://scitation.aip.org/content/aip/journal/jap/117/12?ver=pdfcov

Published by the AIP Publishing

\section{Articles you may be interested in}

Perpendicular-bias-field control of coupled vortex oscillations in nanodot networks

J. Appl. Phys. 117, 083910 (2015); 10.1063/1.4913503

Vortex dynamics in triangular-shaped confining potentials

J. Appl. Phys. 112, 063916 (2012); 10.1063/1.4754418

Magnetisation reversal in permalloy nanowires controlled by near-field charge interactions

Appl. Phys. Lett. 99, 142506 (2011); 10.1063/1.3646548

Precessing vortices and antivortices in ferromagnetic elements

J. Appl. Phys. 109, 023911 (2011); 10.1063/1.3534006

Magnon modes for thin circular vortex-state magnetic dots

Appl. Phys. Lett. 81, 1261 (2002); 10.1063/1.1499515

MIT LINCOLN

LABORATORY CAREERS

Discover the satisfaction of innovation and service

to the nation
- Space Control

- Air \& Missile Defense

- Communications Systems \& Cyber Security

- Intelligence, Surveillance and

Reconnaissance Systems

- Advanced
Electronics
- Tactical Systems
- Homeland
Protection
- Air Traffic Control

LINCOLN LABORATORY

MassachusetTs Institute of TeChNOLOgY

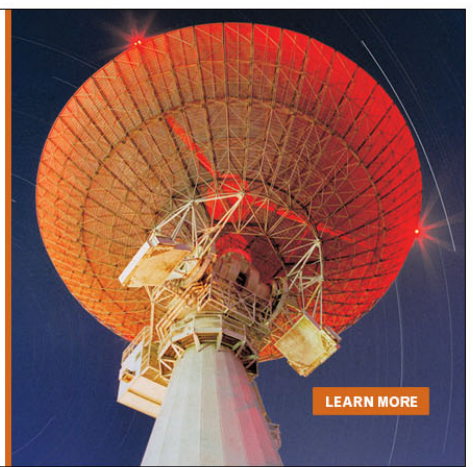




\title{
A comparison of numerical simulations and analytical theory of the dynamics of interacting magnetic vortices
}

\author{
Martin Asmat-Uceda, ${ }^{1}$ Xuemei Cheng, ${ }^{2}$ Xiao Wang, ${ }^{2}$ David J. Clarke,${ }^{3}$ Oleg Tchernyshyov, ${ }^{4}$ \\ and Kristen S. Buchanan ${ }^{1, a)}$ \\ ${ }^{1}$ Department of Physics, Colorado State University, Fort Collins, Colorado 80523, USA \\ ${ }^{2}$ Department of Physics, Bryn Mawr College, Bryn Mawr, Pennsylvania 19010, USA \\ ${ }^{3}$ Department of Physics, California Institute of Technology, Pasadena, California 91125, USA \\ ${ }^{4}$ Department of Physics and Astronomy, Johns Hopkins University, Baltimore, Maryland 20723, USA
}

(Received 12 October 2014; accepted 20 March 2015; published online 31 March 2015)

\begin{abstract}
Magnetostatic interactions between vortices in closely spaced planar structures are important for applications including vortex-based magnonic crystals and spin torque oscillator networks. Analytical theories that include magnetostatic interaction effects have been proposed but have not yet been rigorously tested. Here, we compare micromagnetic simulations of the dynamics of magnetic vortices confined in three disks in an equilateral triangle configuration to analytical theories that include coupling. Micromagnetic simulations show that the magnetostatic coupling between the disks leads to splitting of the gyrotropic resonance into three modes and that the frequency splitting increases with decreasing separation. The temporal profiles of the magnetization depend on the vortex polarities and chiralities; however, the frequencies depend only on the polarity combinations and will fall into one of two categories: all polarities equal or one polarity opposite to the others, where the latter leads to a larger frequency splitting. Although the magnitude of the splitting observed in the simulations is larger than what is expected based on purely dipolar interactions, a simple analytical model that assumes dipole-dipole coupling captures the functional form of the frequency splitting and the motion patterns just as well as more complex models.
\end{abstract} (C) 2015 AIP Publishing LLC. [http://dx.doi.org/10.1063/1.4916610]

\section{INTRODUCTION}

Magnetic vortices, which involve the curling of the magnetic spins into a circular pattern with a central core that points out-of-plane, are often found in micron- and submicron-sized structures that are magnetically soft. ${ }^{1,2}$ Vortices can interact dynamically with one another when confined in the same structure ${ }^{3}$ or in structures that are in close proximity, where coupling in the latter case occurs via magnetostatic interactions. ${ }^{4-7}$ Modifications of the gyrotropic resonance frequency, first predicted to arise in a 2D square array of magnetic vortices, ${ }^{8-10}$ have been observed experimentally for $2 \mathrm{D}$ square arrays $4,11,12$ and in the simpler case of a pair of closely spaced magnetic squares. ${ }^{5,13}$ In the case of the $2 \mathrm{D}$ arrays, the effect is primarily a line-broadening effect of $\sim 20 \%$ for an inter-disk spacing of $20 \%$ of the disk radius, ${ }^{4}$ whereas the gyrotropic resonance frequency splits into two distinct modes for a vortex pair, the frequencies of which depend on the vortex polarities. ${ }^{5}$ The frequency splitting tends to be more pronounced when the polarities are opposite to one another. Coupling effects are important for a variety of applications including vortex-based magnonics, ${ }^{8}$ for increasing the signal from vortex-based spin torque oscillators, ${ }^{14,15}$ and they may also lead to new devices since it was recently shown that the dependence of the resonance frequencies on the polarities can be used to dynamically control and read the state of coupled vortices. ${ }^{16}$ Furthermore, recent studies have shown that

\footnotetext{
a) Author to whom correspondence should be addressed. Electronic mail: Kristen.Buchanan@colostate.edu
}

signals can be transmitted from one disk to another via dipolar coupling in chains of two, ${ }^{5,13,17}$ three, ${ }^{18}$ or more structures lined up in a row, ${ }^{19}$ and schemes to improve the signal transfer rate ${ }^{20}$ and coupling efficiency in vortex chains have been investigated. ${ }^{21}$ Several theories of vortex coupling have been proposed $^{8-10,22,23}$ but have not yet been compared against full micromagnetic simulations.

Here, we conduct a detailed comparison of analytical theory of vortex dynamics including magnetostatic interaction effects with full micromagnetic simulations to determine the extent that the analytical expressions can be used to predict the behavior of interacting vortices. We use a geometry involving three disks arranged to form an equilateral triangle for this comparison, a configuration that is simple enough that it can be treated analytically and realized experimentally but complex enough to serve as a useful test. The magnetization signals are found to exhibit beating patterns in the time domain that vary with the polarities $p$ and chiralities $\chi$; however, the resonance frequencies only depend on whether all $p$ are the same or whether one $p$ differs from the others. The qualitative aspects of the simulations agree well with even the simplest analytical calculations of the dynamics based on purely dipolar interactions; however, more exact models of the dynamic vortex spin distributions will be required to accurately predict the magnitude of the splitting. Our recent time-resolved photo-emission electron microscopy results verify that the dipolar interactions between the vortices are strong enough to cause a splitting in the frequency spectrum that is consistent with the theory and simulation results. ${ }^{24}$ 


\section{MICROMAGNETIC SIMULATIONS}

The dynamic behavior of magnetic vortices in a system consisting of three Permalloy disks arranged in an equilateral triangle (inset of Fig. 1) was simulated using the Object Oriented Micromagnetic Framework (OOMMF). ${ }^{25}$ Disks of diameter $2 R=252 \mathrm{~nm}$ were represented using a $2 \mathrm{D}$ mesh with cells of $4 \times 4 \mathrm{~nm}^{2}$ and thickness $t=20 \mathrm{~nm}$. Material parameters typical for bulk Permalloy were used: exchange stiffness constant of $A=1.3 \times 10^{-11} \mathrm{~J} / \mathrm{m}$, saturation magnetization $M_{s}=8.6 \times 10^{5} \mathrm{~A} / \mathrm{m}$, gyromagnetic ratio $\gamma=1.76 \times 10^{11} \mathrm{~s}^{-1} \mathrm{~T}^{-1}$, and the magnetocrystalline anisotropy was neglected. A variety of vortex $\chi$ and $p$ combinations were considered for a fixed radius $R$ to inter-disk center-to-center separation $D$ ratio of $R / D=0.45$. The dependence of the mode frequencies on $D$ was simulated for selected $\chi$ and $p$ combinations for $R / D$ from 0.20 to 0.49 , which covers the range from where the disks are far enough apart that the magnetostatic interactions are small to where the disks are almost touching. The magnetization was first allowed to relax to an equilibrium configuration in the presence of a static field of $\mu_{o} H=5 \mathrm{mT}$ applied along the direction connecting two disks centers ( $x$-direction) using a large damping parameter $\alpha=1$ for fast convergence, and then the static field was abruptly removed and the dynamics were modeled using a realistic $\alpha=0.01$. Additional simulations were conducted for selected configurations with $\alpha=0.001$ to construct spatial profiles of the individual eigenmodes.

Fig. 1 shows the time-evolution of the magnetization of each disk and the corresponding Fourier spectra for a variety of $p$ combinations and fixed, identical $\chi$ for $R / D=0.45$. The details of the temporal responses vary with the choice of $p$; however, two main types of responses were observed: those associated with disks of all the same $p$ and those with one $p$ reversed. When $\chi$ and $p$ are all the same (Figs. 1(a) and $1(\mathrm{e})$ ), two frequencies are observed: a dominant resonance at $630 \mathrm{MHz}$ and a weaker resonance at $690 \mathrm{MHz}$, which both differ from the single vortex gyrotropic frequency of
$680 \mathrm{MHz}$ (not shown). The same spectral response and overall temporal characteristics are present for other $\chi$ combinations as well (not shown). In contrast, when one $p$ is opposite to the others, three distinct resonant frequencies are observed at 600,660 , and $750 \mathrm{MHz}$ (Figs. 1(b)-1(d) and 1(f)-1(h)) and pronounced beating patterns are detected in the time domain. The phases and the relative mode amplitudes and hence the details of the temporal patterns depend on the exact $p$ and $\chi$ configuration, but the frequencies depend only on the relative $p$ of the three cores.

Figure 2 shows how resonance frequencies change as a function of $D$. When all vortices have the same $p$ (Fig. 2(a)), the resonance splits into two different modes as the disks are brought closer together, whereas when one $p$ is opposite to the others, three different frequencies are observed (Fig. 2(b)). In both cases, the magnitude of the splitting increases with $D$ up to a maximum shift of $22 \%(R / D=0.49)$. When the disks are further apart $(R / D=0.20)$, the vortices oscillate at the single disk gyrotropic frequency of $680 \mathrm{MHz}$.

\section{ANALYTICAL CALCULATIONS}

From an analytical perspective, the Thiele equation, which describes the dynamics of a magnetic vortex in terms of the position of its core, serves as the basis for most models of vortex dynamics. ${ }^{26}$ Theoretical models have been developed to describe the expected modes for vortices coupled via the magnetostatic fields that are generated by the moving vortices for a variety of geometries. Calculations have been conducted for vortices in arranged in a 2D square array, ${ }^{8}$ for the normal modes for two disks that are excited together, ${ }^{23}$ and a more general treatment examines vortex pairs as well as 2D square and hexagonal arrays. ${ }^{22}$ The main focus of the previous work has been on the calculation of interaction integrals for specific models of the vortex spin distribution, e.g., the poles-free model. ${ }^{22}$ Here, we derive the theory of the expected modes for the triangular arrangement considered in the simulations using a simple dipole-dipole
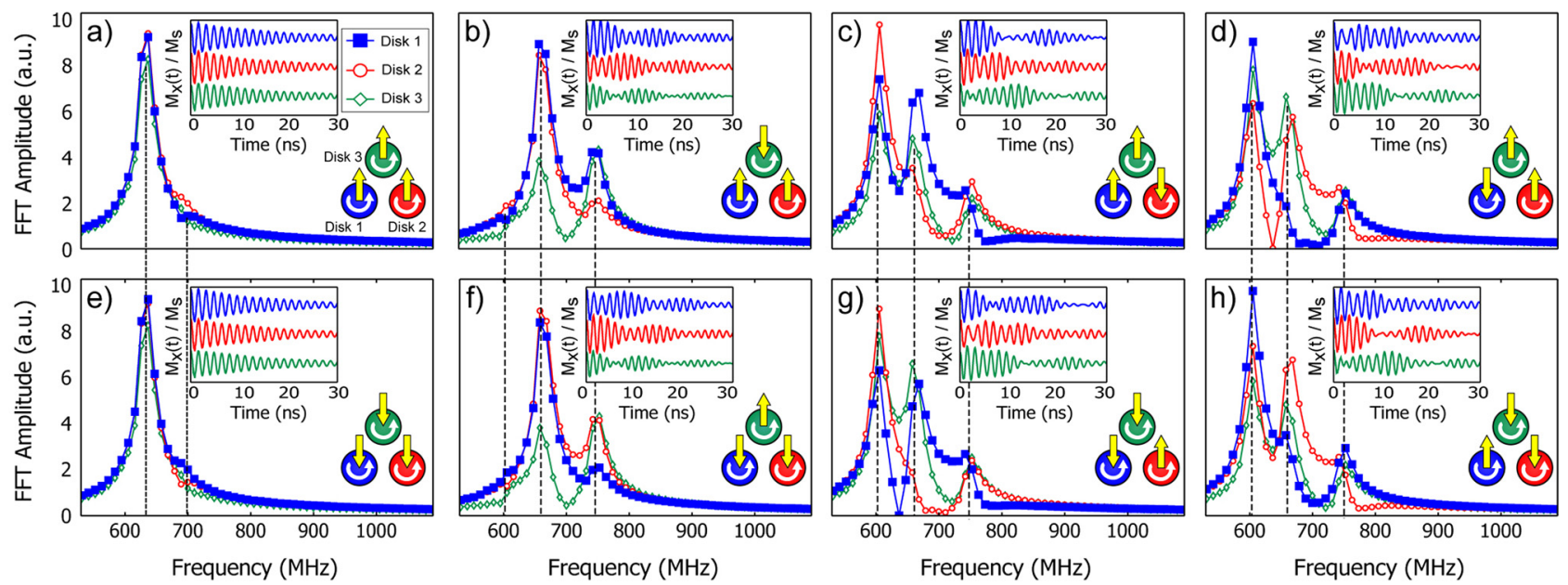

FIG. 1. Micromagnetic simulations of three interacting disks with $R / D=0.45$ for a variety of $p$ (vertical arrows) and fixed, identical $\chi$ (curved arrows). Fourier transforms of the $x$-component of the volume-average magnetization $M_{x}$ of each disk are shown. The insets show $M_{x}$ as a function of time. In all cases, the maximum amplitude of the oscillations is $M_{x} / M_{s} \sim 0.1$. When $p$ are (a) all up and (e) all down, a strong resonance at $630 \mathrm{MHz}$ as well as a weaker resonance at $690 \mathrm{MHz}$ are observed, whereas three resonances of 600,660 , and $750 \mathrm{MHz}$ are observed when one core is down and the others are up ((b), (c), and (d)) or one is up and two are down ((f), (g), and (h)). 

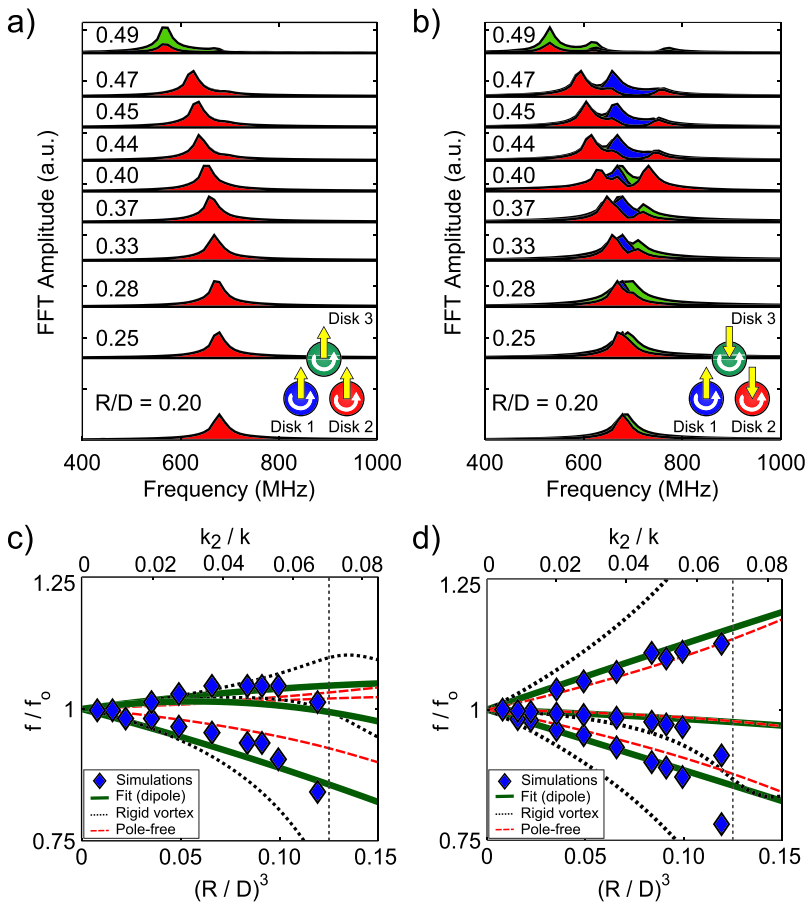

FIG. 2. The Fourier spectra for each disk are shown for a range of $D$ for the cases when (a) all vortices have the same core polarities $(p=+1)$, and in (b), there is one opposing polarity $(p=-1)$. The spectra baselines are shifted by an amount proportional to $D$. The corresponding resonant frequencies from simulations (symbols) and theory (lines) are shown as a function of $(R / D)^{3}$ in (c) and (d), where (c) are for $p=1$ and (d) are for $p=-1$. The solid green line represents the dipole-dipole interaction model where $C_{f i t}=2.82 \pm 0.06$ was used as the only fitting parameter. The disks are in contact for $R / D \geq 0.5$ (vertical dotted line).

interaction model and compare the eigenfrequencies and eigenvalues to the simulation results.

For small displacements of a vortex $\overrightarrow{\mathrm{x}}$ from its central equilibrium position, there is a restoring force $\overrightarrow{\mathrm{F}}=-k \overrightarrow{\mathrm{x}}$ on the vortex that is mainly due to the increase in the demagnetization energy. Interplay between the restoring and gyrotropic forces gives rise to spiraling motion with a characteristic resonance frequency $\omega_{\mathrm{o}}=k / G$, where $G=2 \pi t M_{s} / \gamma$ is the gyrotropic constant that is independent of $p= \pm 1$ and $\chi= \pm 1$, where $\chi=+1$ indicates a counterclockwise vortex. Reference 27 gives an expression for $\omega_{0}$ in terms of the magnetic and geometrical parameters of the disk. In the limit of small aspect ratio $\beta=t / R \ll 1, \omega_{\mathrm{o}}=\frac{5}{9 \pi} \gamma \mu_{o} M_{s} \beta$, where $\mu_{o}$ is the magnetic permeability of free space, which corresponds to $k_{\text {approx }}=\frac{10 \mu_{o} M_{s}^{2} t^{2}}{9 R}$. This estimate is reasonable for $\beta<0.1$; for the geometry used here, $\beta=0.16$, so we have calculated $k$ using the full expressions given in Ref. 27, which yields $k=0.81 \cdot k_{\text {approx }}$.

Magnetic vortices in adjacent disks will be coupled via magnetostatic interactions, and the dominant term in a multipole expansion will be the dipole-dipole interaction term. The interactions are generally only significant when the space between the disks is considerably smaller than the disk diameter, and in this case, higher order terms in a multipole expansion are needed to fully describe the magnetostatic interactions. $^{22}$ Nevertheless, as we will show below, the expressions obtained using just the dipole-dipole interactions are sufficient to describe the eigenfrequencies as a function of the inter-disk separation and the eigenvalues. For small displacements, the dipole moment $\vec{d}$ of a shifted vortex can be approximated as

$$
\overrightarrow{\mathrm{d}}=-C \chi M_{s} t R \hat{\mathrm{z}} \times \overrightarrow{\mathrm{x}}
$$

where dimensionless factor $C$ is a function of $t / R$. The dipole-dipole interaction energy between the disks is

$$
E_{\text {dip }}=\frac{\mu_{o}}{4 \pi \mathrm{D}_{\mathrm{ij}}^{3}}\left(\overrightarrow{\mathrm{d}}_{\mathrm{i}} \cdot \overrightarrow{\mathrm{d}}_{\mathrm{j}}-3\left(\hat{\mathrm{D}}_{\mathrm{ij}} \cdot \overrightarrow{\mathrm{d}}_{\mathrm{i}}\right)\left(\hat{\mathrm{D}}_{\mathrm{ij}} \cdot \overrightarrow{\mathrm{d}}_{\mathrm{j}}\right)\right),
$$

where $\hat{D}_{i j}$ is the unit vector pointing from the center of disk $i$ to the center of disk $\mathrm{j}$, which can be written in terms of the vortex displacements as

$$
\mathrm{E}_{\mathrm{dip}}=k_{2} \chi_{\mathrm{i}} \chi_{\mathrm{j}}\left(\overrightarrow{\mathrm{x}}_{\mathrm{i}} \cdot \overrightarrow{\mathrm{x}}_{\mathrm{j}}-3\left(\hat{\mathrm{z}} \cdot \hat{\mathrm{D}}_{\mathrm{ij}} \times \overrightarrow{\mathrm{x}}_{\mathrm{i}}\right)\left(\hat{\mathrm{z}} \cdot \hat{\mathrm{D}}_{\mathrm{ij}} \times \overrightarrow{\mathrm{x}}_{\mathrm{j}}\right)\right)
$$

where $k_{2}=C^{2} \mu_{o} M_{s}^{2} t^{2} R^{2} / 4 \pi D^{3}$ for circular disks. For two disks arranged along the $\mathrm{x}$-axis, this reduces to $\mathrm{E}_{\mathrm{int}}=\chi_{1} \chi_{2}$ $\left(k_{2} \mathrm{x}_{1} \mathrm{x}_{2}-2 k_{2} \mathrm{y}_{1} \mathrm{y}_{2}\right)$. This can be written more generally in the form $\mathrm{E}_{\mathrm{int}}=\chi_{1} \chi_{2}\left(n_{x} \mathrm{x}_{1} \mathrm{x}_{2}+n_{y} \mathrm{y}_{1} \mathrm{y}_{2}+O\left(\left|\overrightarrow{\mathrm{x}}_{\mathrm{j}}\right|^{3}\right)\right)$, as in Ref. 22 , where $n_{x}$ and $n_{y}$ are interaction coefficients that can be calculated for not just dipolar but also higher order magnetostatic interaction effects. For purely dipolar interactions, $n_{x}=k_{2}$ and $n_{y}=-2 k_{2}$. Methods for calculating the coefficients $n_{x}$ and $n_{y}$ for two specific theoretical models of the vortex spin distribution, the rigid vortex model and the poles-free model, are given in Ref. 22 (see Eq. (13)) where the interaction energies are integrated over the full vortex magnetization distributions. Regardless of the form of the interaction energy, the Lagrangian for an ensemble of disks is given by

$$
\mathrm{L}=\sum_{\mathrm{i}}\left[\left(-\mathrm{p}_{\mathrm{i}}\right) \mathrm{G} \dot{\mathrm{x}}_{\mathrm{i}} \mathrm{y}_{\mathrm{i}}-\frac{\mathrm{k}}{2}\left|\overrightarrow{\mathrm{x}}_{i}\right|^{2}\right]-\sum_{\mathrm{i}<j} \mathrm{E}_{\mathrm{int}}^{\mathrm{ij}} .
$$

We can remove the chiralities by switching variables to $\overrightarrow{\mathrm{x}}_{\mathrm{i}} \rightarrow \chi_{\mathrm{i}} \overrightarrow{\mathrm{x}}_{\mathrm{i}}$, which means that the frequencies are independent of $\chi_{\mathrm{i}}$. They will, however, depend on $p$.

As mentioned above, there are only two possible spectra based on the vortex polarities. Without loss of generality, we shall set $p_{1}=p_{2}=1$, and leave $p_{3}=p$ to distinguish the two possibilities. Setting disks 1 and 2 along the $x$-axis, the magnetostatic energy of the system is

$$
\begin{aligned}
\mathrm{E}_{\text {int }}= & n_{x} \mathrm{x}_{1} \mathrm{x}_{2}+n_{y} \mathrm{y}_{1} \mathrm{y}_{2}+\frac{1}{4}\left(n_{x}+3 n_{y}\right)\left(\mathrm{x}_{1}+\mathrm{x}_{2}\right) \mathrm{x}_{3} \\
& +\frac{1}{4}\left(3 n_{x}+n_{y}\right)\left(\mathrm{y}_{1}+\mathrm{y}_{2}\right) \mathrm{y}_{3}-\frac{\sqrt{3}}{4}\left(n_{x}-n_{y}\right)\left(\mathrm{x}_{2}-\mathrm{x}_{1}\right) \mathrm{y}_{3} \\
& -\frac{\sqrt{3}}{4}\left(n_{x}-n_{y}\right)\left(\mathrm{y}_{2}-\mathrm{y}_{1}\right) \mathrm{x}_{3} .
\end{aligned}
$$

If we define new coordinates $\overline{\mathrm{x}}=\left(\mathrm{x}_{1}+\mathrm{x}_{2}\right) / \sqrt{2}, \overline{\mathrm{y}}$ $=\left(\mathrm{y}_{1}+\mathrm{y}_{2}\right) / \sqrt{2}, \mathrm{~d}=\left(\mathrm{x}_{1}-\mathrm{x}_{2}\right) / \sqrt{2}$, and $\Delta=\left(\mathrm{y}_{1}-\mathrm{y}_{2}\right) / \sqrt{2}$, then the equations of motion are given by 


$$
\begin{gathered}
G\left(\begin{array}{c}
\dot{\bar{y}} \\
\dot{y}_{3} \\
\dot{d}
\end{array}\right)=\left(\begin{array}{ccc}
\left(k+n_{x}\right) & \frac{\sqrt{2}}{4}\left(n_{x}+3 n_{y}\right) & 0 \\
p \frac{\sqrt{2}}{4}\left(n_{x}+3 n_{y}\right) & p k & p \frac{\sqrt{6}}{4}\left(n_{x}-n_{y}\right) \\
0 & -\frac{\sqrt{6}}{4}\left(n_{x}-n_{y}\right) & -\left(k-n_{y}\right)
\end{array}\right)\left(\begin{array}{c}
\bar{x} \\
x_{3} \\
\Delta
\end{array}\right), \\
G\left(\begin{array}{c}
\dot{\bar{x}} \\
\dot{x}_{3} \\
\dot{\Delta}
\end{array}\right)=\left(\begin{array}{ccc}
-\left(k+n_{y}\right) & -\frac{\sqrt{2}}{4}\left(3 n_{x}+n_{y}\right) & 0 \\
-p \frac{\sqrt{2}}{4}\left(3 n_{x}+n_{y}\right) & -p k & -p \frac{\sqrt{6}}{4}\left(n_{x}-n_{y}\right) \\
0 & \frac{\sqrt{6}}{4}\left(n_{x}-n_{y}\right) & \left(k-n_{x}\right)
\end{array}\right)\left(\begin{array}{c}
\bar{y} \\
y_{3} \\
\mathrm{~d}
\end{array}\right) .
\end{gathered}
$$

In the form where only dipolar coupling is considered, the equations of motion reduce to

$$
\begin{aligned}
G\left(\begin{array}{c}
\dot{\bar{y}} \\
\dot{y}_{3} \\
\dot{d}
\end{array}\right)=\left(\begin{array}{ccc}
\left(k+k_{2}\right) & -\frac{5 \sqrt{2}}{4} k_{2} & 0 \\
-p \frac{5 \sqrt{2}}{4} k_{2} & p k & p \frac{3 \sqrt{6}}{4} k_{2} \\
0 & -\frac{3 \sqrt{6}}{4} k_{2} & -\left(k+2 k_{2}\right)
\end{array}\right)\left(\begin{array}{c}
\bar{x} \\
x_{3} \\
\Delta
\end{array}\right), \\
G\left(\begin{array}{c}
\dot{\bar{x}} \\
\dot{x}_{3} \\
\dot{\Delta}
\end{array}\right)=\left(\begin{array}{ccc}
-\left(k-2 k_{2}\right) & -\frac{\sqrt{2}}{4} k_{2} & 0 \\
-p \frac{\sqrt{2}}{4} k_{2} & -p k & -p \frac{3 \sqrt{6}}{4} k_{2} \\
0 & \frac{3 \sqrt{6}}{4} k_{2} & \left(k-k_{2}\right)
\end{array}\right)\left(\begin{array}{c}
\bar{y} \\
y_{3} \\
\mathrm{~d}
\end{array}\right) .
\end{aligned}
$$

The eigenfrequencies and eigenvalues can be obtained numerically from Eq. (6) or (7). These expressions are valid for any structure shape, not just circular disks, provided that the values for $k_{2}$ (or $n_{x}$ and $n_{y}$ ), which can be derived for just a pair of disks, and $k$, which pertains to a single structure are obtained for the geometry of interest. These parameters do depend on the structure shape and dimensions and can be found either through the evaluation of appropriate interaction integrals or from simulations of just one or two disks. Furthermore, the method used to derive (6) and (7) can be easily adapted to any geometrical arrangement of an arbitrary number of structures.

The dependence of the eigenfrequencies of (6) on $k_{2} / k$ are shown in Figs. 2(c) and 2(d), where $\frac{k_{2}}{k}=\frac{9 C^{2}}{40 \pi}\left(\frac{R}{D}\right)^{3}$ (valid for circular disks). Note that the solutions are not all real for all $k_{2} / k$. For $k_{2} / k>2 / 5$, at least one frequency is imaginary, suggesting that the vortices are destabilized. In Figs. 2(c) and 2(d), the solid green line shows the dipole-dipole interaction results using a value $C_{f i t}=2.82 \pm 0.06$ that was extracted from simultaneous fits to the results shown in both plots (where the full expression in Ref. 27 was used to find $k$ ). This
$C_{\text {fit }}$ yields $k_{2} / k \approx 0.070$ for the maximum $R / D=0.5$ (disks touching), which is below the instability threshold. Note that the resonance frequencies obtained for $R / D=0.49$ were not included in the fits since the gap between the disks is as little as a single cell and edge effects may artificially increase the coupling effects; for $R / D=0.44$, a $<2 \%$ difference was observed when the cell size used in the simulations was reduced from $4 \times 4 \mathrm{~nm}^{2}$ to $2 \times 2 \mathrm{~nm}^{2}$.

\section{RESULTS AND DISCUSSION}

The frequency spectra obtained from the simulations agree well with the theory (Eq. (7)), provided $C$ is used as a fitting parameter (Figs. 2(c) and 2(d)). Equations (6) and (7) predict three distinct modes for both $p$ combinations; however, when all $p$ are the same, the two higher-frequency modes are quite close, which is why only two modes are identified in the simulations. The frequency splitting is more pronounced for $p=-1$. The modes become distinguishable from one another at slightly lower $R / D$ (larger $D$ ), and the magnitude of the splitting is larger, which is consistent with reports of stronger coupling for opposing polarities in experiments ${ }^{19}$ and simulations. ${ }^{20}$

There are, however, discrepancies in the predicted magnitude of the splitting effects for all of the models considered. In Fig. 2, the parameter $C$ was used as a fitting parameter to set the scale of the dipolar interaction model; however, its value can be estimated from the simulations using Eq. (1) and fits to the core positions and magnetization values. This yields $C_{d i p}=2.0 \pm 0.1$, which is smaller than the value obtained from fitting, which was $C_{f i t}=2.82 \pm 0.06$, hence the dipole model underestimates the magnitude of the observed splitting. Figs. 2(c) and 2(d) also show the frequency splitting that is predicted from Eq. (6) using $n_{x}$ and $n_{y}$ for the two-vortex and poles-free models from Ref. 22, which include higher order interaction effects. The poles-free model provides closer agreement but still underestimates the splitting, whereas the rigid vortex model overestimates the effect. The functional form of $f / f_{o}$ vs. $(R / D)^{3}$ is similar for all of the models, which occurs because the ratio $n_{x} / n_{y}$ is close to the dipolar ratio of $-1 / 2$. The simple 
a)
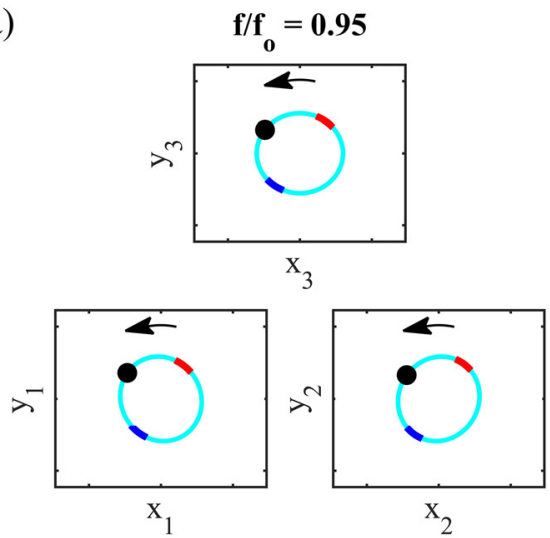

c)

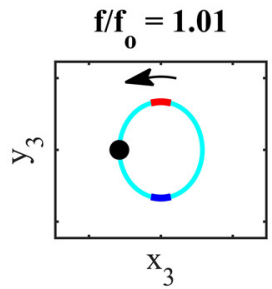

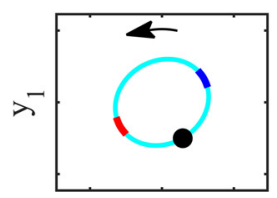

$\mathrm{x}_{1}$

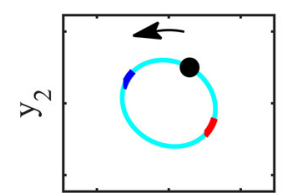

$\mathrm{x}_{2}$ e)
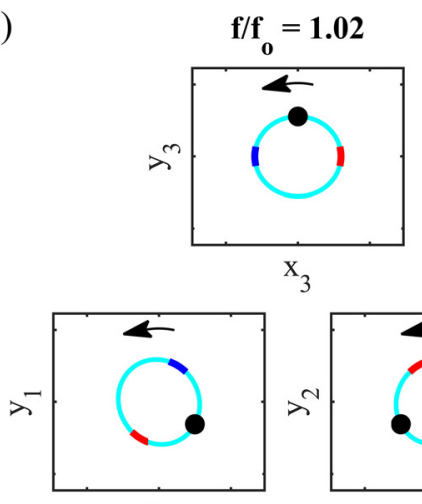

$\mathrm{X}_{1}$

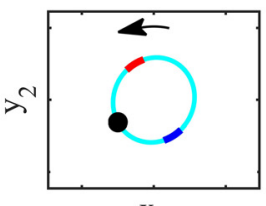

b)
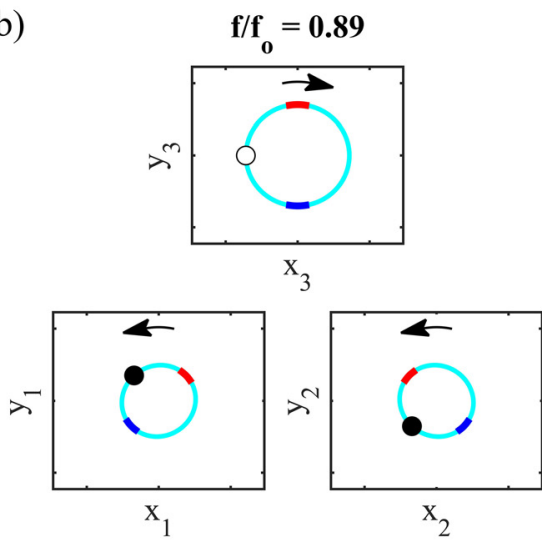

d)

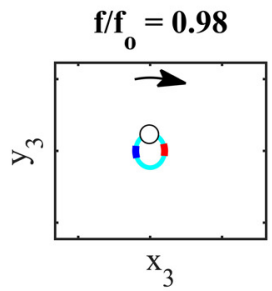

$x_{3}$

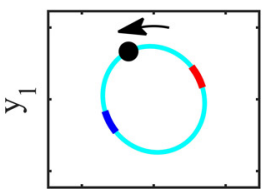

$x_{1}$

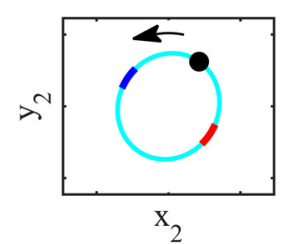

$\mathbf{f} / \mathbf{f}_{0}=1.11$
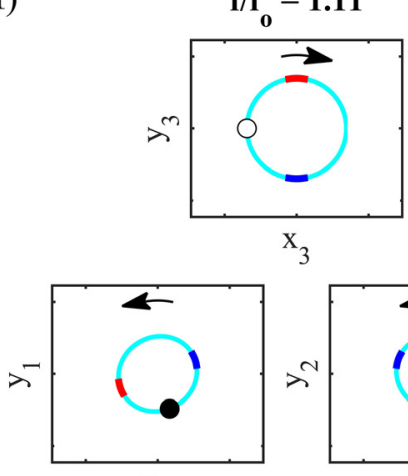

$\mathrm{X}_{1}$

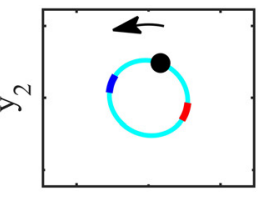

$\mathrm{x}_{2}$
FIG. 3. Illustration of the eigenmodes of Eq. (6) for $R / D=0.45$. The solid (cyan) ellipses show the core trajectories for each disk. The core at an instant of time is indicated by a black (white) circle for a positive (negative) $p$, and the arrows indicate the core's direction of travel. The dipole moments that develop when the vortices are displaced from equilibrium are illustrated by the red and blue shaded regions that represent the north and south poles, respectively. Counterclockwise $\chi$ are assumed for all vortices. (Multimedia view) [URL: http://dx.doi.org/10.1063/ $1.4916610 .1]$ dipolar model therefore provides a useful functional form for the splitting; however, an intermediate model for the magnetization profile of a dynamic vortex is needed to correctly predict the magnitude of the effect.

Fig. 3 (Multimedia view) illustrates the normal modes for the system from Eq. (6). Similar results are obtained for the poles-free and rigid vortex models, again because $n_{x} / n_{y}$ $\sim-1 / 2$. Figs. 3(a), 3(c), and 3(e) show the modes for $p=1$. For the lowest frequency mode (Fig. 3(a)), the cores all have close to the same phase at any given time and attractive interactions occur whenever the dipoles are aligned along an axis that connects two disk centers. A spatially uniform field pulse applied to disks with the same $\chi$ provides a phase relationship between the cores that is similar to this mode, hence this is the dominant mode in Fig. 2(a). When the core in the top disk is reversed (Fig. 3(b)), there are still attractive interactions for lowest frequency mode but the phases are timed such that when the north or south pole of the top disk is at its lowest point it is attracted equally to both of the lower disks.

In contrast, the higher frequency modes involve mainly repulsive interactions or combinations of repulsion and attraction. Repulsive interactions occur for the two higher frequency modes for $p=1$ (Figs. 3(c) and 3(e)) but at different phases for each. For $p=-1$, the middle mode (Fig. 3(d)) involves motion of mainly the two bottom vortices and is characterized by both attractive and repulsive interactions, whereas the highest frequency mode (Fig. 3(f)) mainly involves repulsive interactions that occur when like dipoles are aligned along an axis that connects two disk centers.

Spatial profiles of the normal modes (Fig. 4 shows modes for $p=-1$ ) were obtained from the simulations by calculating the Fourier transform of the $z$-component of the magnetization for each cell, as described in Ref. 28. Plots of $A \cos \phi$, where $A$ and $\phi$ are the amplitude and phase, respectively, calculated from the simulations for $R / D=0.45$ 
a)

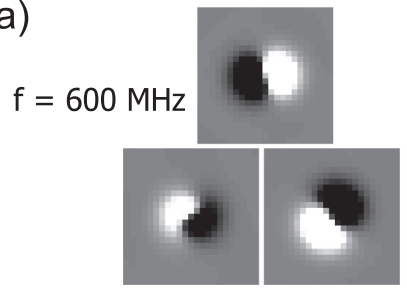

b)

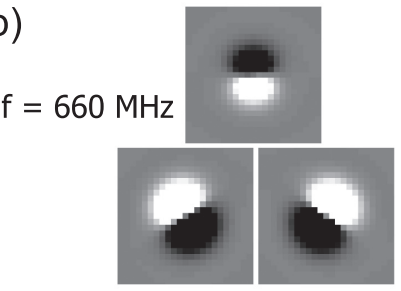

c)

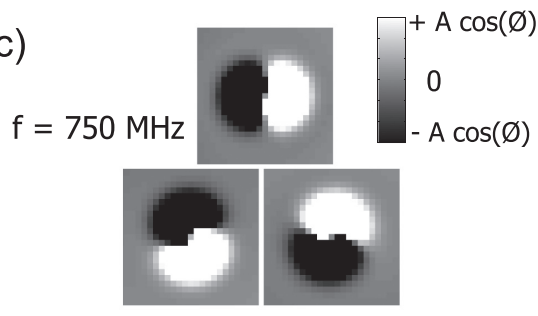

FIG. 4. Mode maps obtained from simulations for $R / D=0.45$ with $p=-1$ where the top disk has a negative core polarity, which is the configuration shown in Figs. 3(b), 3(d), and 3(f). Each plot represents an area of $93 \times 93 \mathrm{~nm}^{2}$. All of the vortices have counterclockwise $\chi$.

provide a snapshot of the core positions in each disk. The phase has been shifted so that white (black) coincides with the positive (negative) pole of the dipole in the theoretical modes shown in Figs. 3(b), 3(d), and 3(f) to show that the relative phases observed in the simulations agree well with the eigenmodes of Eq. (6). Similar agreement is observed for the low frequency $p=1$ mode (Fig. 3(a)), but it is difficult to obtain reliable mode maps for the two higher frequency $p=1$ modes because they are so close in frequency.

\section{CONCLUSIONS}

In conclusion, we have compared results from micromagnetic simulations and analytical theory for a system of interacting vortices in three magnetic disks arranged in an equilateral triangle. The theory and simulations agree on the main features of the observed dynamics. The vortex gyrotropic mode splits into three modes, the magnitude of the frequency splitting increases with decreasing $D$, and only two distinct frequency splitting behaviors are observed that depend on whether all $p$ are the same or one $p$ opposes the others, where the latter leads to stronger splitting. A simple model involving only dipolar interactions accurately captures the qualitative features of the coupled vortex dynamics including the frequencies and eigenmodes; however, a more detailed model of the dynamic vortex spin distribution will be required to improve predictions of the magnitude of the frequency splitting. Nevertheless, the simple dipole-dipole model, once calibrated for a particular disk geometry, provides an excellent description of the dynamics of interacting vortices that can be extended to more complex arrangements of magnetic vortices, including square or hexagonal arrays and chains of arbitrary geometries, which will be helpful for the development and optimization of new vortex-based devices.

\section{ACKNOWLEDGMENTS}

This work was supported by the NSF under Grant Nos. NSF DMR-0907706 (CSU), NSF DMR-1053854 (Bryn Mawr), and NSF DMR-1104753 (Johns Hopkins); work at the Advanced Photon Source was supported by the U.S. DOE-BES, Contract No. DE-AC02-06CH11357.

${ }^{1}$ T. Shinjo, T. Okuno, R. Hassdorf, K. Shigeto, and T. Ono, Science 289, 930-932 (2000).
${ }^{2}$ R. P. Cowburn, D. K. Koltsov, A. O. Adeyeye, M. E. Welland, and D. M. Tricker, Phys. Rev. Lett. 83, 1042-1045 (1999).

${ }^{3}$ K. S. Buchanan, P. E. Roy, M. Grimsditch, F. Y. Fradin, K. Y. Guslienko, S. D. Bader, and V. Novosad, Nat. Phys. 1, 172-176 (2005).

${ }^{4}$ A. Vogel, A. Drews, T. Kamionka, M. Bolte, and G. Meier, Phys. Rev. Lett. 105, 037201 (2010).

${ }^{5}$ S. Sugimoto, Y. Fukuma, S. Kasai, T. Kimura, A. Barman, and Y. Otani, Phys. Rev. Lett. 106, 197203 (2011).

${ }^{6}$ K. Arai, T. Okuda, K. Fukumoto, M. Kotsugi, T. Ohkouchi, K. Kodama, T. Kimura, Y. Haruyama, T. Nakamura, T. Matsushita, H. Osawa, T. Muro, S. Matsui, A. Kakizaki, Y. Otani, and T. Kinoshita, Jpn. J. Appl. Phys. 50, 053001 (2011).

${ }^{7}$ K.-S. Lee, H. Jung, D.-S. Han, and S.-K. Kim, J. Appl. Phys. 110, 113903 (2011).

${ }^{8}$ J. Shibata, K. Shigeto, and Y. Otani, Phys. Rev. B 67, 224404 (2003).

${ }^{9}$ J. Shibata and Y. Otani, Phys. Rev. B 70, 012404 (2004).

${ }^{10}$ A. Y. Galkin, B. A. Ivanov, and C. E. Zaspel, Phys. Rev. B 74, 144419 (2006).

${ }^{11}$ A. Barman, S. Barman, T. Kimura, Y. Fukuma, and Y. Otani, J. Phys. D: Appl. Phys. 43, 422001 (2010).

${ }^{12}$ A. A. Awad, G. R. Aranda, D. Dieleman, K. Y. Guslienko, G. N. Kakazei, B. A. Ivanov, and F. G. Aliev, Appl. Phys. Lett. 97, 132501 (2010).

${ }^{13}$ A. Vogel, T. Kamionka, M. Martens, A. Drews, K. W. Chou, T. Tyliszczak, H. Stoll, B. Van Waeyenberge, and G. Meier, Phys. Rev. Lett. 106, 137201 (2011).

${ }^{14}$ V. S. Pribiag, I. N. Krivorotov, G. D. Fuchs, P. M. Braganca, O. Ozatay, J. C. Sankey, D. C. Ralph, and R. A. Buhrman, Nat. Phys. 3, 498-503 (2007).

${ }^{15}$ N. Locatelli, V. V. Naletov, J. Grollier, G. D. Loubens, V. Cros, C. Deranlot, C. Ulysse, G. Faini, O. Klein, and A. Fert, Appl. Phys. Lett. 98, 062501 (2011).

${ }^{16}$ S. Jain, V. Novosad, F. Y. Fradin, J. E. Pearson, V. Tiberkevich, A. N. Slavin, and S. D. Bader, Nat. Commun. 3, 1330 (2012).

${ }^{17}$ H. Jung, Y.-S. Yu, K.-S. Lee, M.-Y. Im, P. Fischer, L. Bocklage, A. Vogel, M. Bolte, G. Meier, and S.-K. Kim, Appl. Phys. Lett. 97, 222502-222503 (2010).

${ }^{18}$ A. Vogel, M. Martens, M. Weigand, and G. Meier, Appl. Phys. Lett. 99, 042506 (2011).

${ }^{19}$ D. S. Han, A. Vogel, H. Jung, K. S. Lee, M. Weigand, H. Stoll, G. Schutz, P. Fischer, G. Meier, and S. K. Kim, Sci. Rep. 3, 2262 (2013).

${ }^{20}$ J.-H. Kim, K.-S. Lee, H. Jung, D.-S. Han, and S.-K. Kim, Appl. Phys. Lett. 101, 092403-092404 (2012).

${ }^{21}$ S. Barman, A. Barman, and Y. Otani, IEEE Trans. Magn. 46, 1342-1345 (2010).

${ }^{22}$ O. V. Sukhostavets, J. González, and K. Y. Guslienko, Phys. Rev. B 87, 094402 (2013).

${ }^{23}$ O. V. Sukhostavets, J. M. Gonzalez, and K. Y. Guslienko, Appl. Phys. Express 4, 065003 (2011).

${ }^{24}$ X. Wang, D. J. Keavney, M. Asmat-Uceda, K. S. Buchanan, A. Melikyan, and X. M. Cheng, Appl. Phys. Lett. 105, 102408 (2014).

${ }^{25}$ J. Donahue and D. G. Porter, OOMMF User's guide, Version 1.0, Interagency Report No. NIST IR 6376, Gaithersburg, MD, 1999.

${ }^{26}$ A. A. Thiele, Phys. Rev. Lett. 30, 230-233 (1973).

${ }^{27}$ K. Y. Guslienko, X. F. Han, D. J. Keavney, R. Divan, and S. D. Bader, Phys. Rev. Lett. 96, 067205 (2006).

${ }^{28}$ M. Yan, G. Leaf, H. Kaper, R. Camley, and M. Grimsditch, Phys. Rev. B 73, 014425 (2006). 